\title{
Hypovirulence and Double-Stranded RNA in Sclerotinia homoeocarpa
}

\author{
Ting Zhou and Greg J. Boland
}

First author: Agriculture and Agri-Food Canada, Pest Management Research Centre, 4902 Victoria Ave. N., P.O. Box 6000, Vineland Station, ON, Canada, L0R 2E0; and second author: Department of Environmental Biology, University of Guelph, Guelph, ON, Canada, N1G 2W1. Accepted for publication 28 October 1996.

\begin{abstract}
Zhou, T., and Boland, G. J. 1997. Hypovirulence and double-stranded RNA in Sclerotinia homoeocarpa. Phytopathology 87:147-153.

One hundred and thirty-two isolates of Sclerotinia homoeocarpa, the causal agent of dollar spot of turfgrass, were evaluated for virulence on swards and detached leaves of creeping bentgrass and for the presence of double-stranded RNA (dsRNA). In at least four isolates, the hypovirulent phenotype was associated with the presence of specific segments of dsRNA. In addition, these hypovirulent isolates often grew slowly on potato dextrose agar (PDA), formed thin colonies with atypical colony margins, and failed to produce typical black stroma. The hypovirulent phenotype and dsRNA were transmitted from hypovirulent isolate Sh12B to virulent isolate $\mathrm{Sh} 48 \mathrm{~B}$, and the converted isolate was hypovirulent and

transmitted to at least four other isolates of the pathogen, including the fungicide-resistant, dsRNA- isolate KY-7. Converted isolates of KY-7 developed the hypovirulent phenotype, grew on fungicide-amended medium, and contained dsRNA. Subcultures of hypovirulent isolate Sh12B that did not contain dsRNA were obtained through curative treatment using cycloheximide-containing medium and heat. Cured subcultures grew faster on PDA, had more typical colony morphologies, were more virulent on bentgrass leaves, and did not contain dsRNA. No cured subcultures were obtained from hypovirulent isolate Sh09B. Isolates regenerated from protoplasts of hypovirulent isolate Sh12B were not cured, remained hypovirulent, and contained dsRNA. Transmission of hypovirulence and dsRNA in S. homoeocarpa has potential as a novel approach to the management of dollar spot of turfgrass.
\end{abstract} contained dsRNA. The hypovirulent phenotype and dsRNA also were
Dollar spot disease, caused by Sclerotinia homoeocarpa F. T. Bennett (possibly a Lanzia sp. or Moellerodiscus sp.), is a widespread and destructive disease of many turfgrasses, including cool and warm season species. Strategies for dollar spot management currently rely on applying fungicides, increasing nitrogen fertilization, avoiding drought stress, and avoiding or removing dew and guttation fluid $(33,34)$. However, more efficient and environmentally benign control strategies are needed. Bacteria and fungi antagonistic to dollar spot have been reported $(18,29)$, but have not been used in dollar spot management.

Hypovirulence is a term used to describe the reduced ability to cause disease by selected isolates within a population of fungal pathogens and has been reported for an increasing number of plant pathogens $(4,9,19,27,30,36)$. In Europe and some regions of the United States, hypovirulent isolates of Cryphonectria parasitica have successfully controlled chestnut blight (26). Decline of root disease caused by Rhizoctonia solani also has been associated with hypovirulence $(9,10)$. Hypovirulence is receiving increased attention, because of its potential use in biological control of fungal plant pathogens.

Hypovirulence can have several causes, but is often associated with the presence of mycoviruses or unencapsidated doublestranded RNA (dsRNA) $(4,8,9,14,19,27,30,36)$; some of the latter have been classified within the unique virus family Hypoviridae (20). Considerable diversity exists in the amount, size, and number of segments of dsRNA recovered from individual isolates of C. parasitica $(24,31)$, and relatively little cross-hybridization occurs among isolates from various regions of Europe and North America $(21,24,31)$. Typically, isolates that contain hypovirulence-associated dsRNA have, alone or in combination, reduced

Corresponding authors: G. J. Boland; E-mail address: gboland@evbhort.uoguelph.ca T. Zhou; E-mail address: zhout@em.agr.ca

Publication no. P-1997-0114-02R

(C) 1997 Department of Agriculture and Agri-Food, Government of Canada virulence, altered culture morphology, reduced growth rates, and changes in pigmentation (30). However, not all dsRNA has been associated with detectable alterations in fungal phenotypes. In $C$. parasitica, a 12-kb segment of dsRNA present in $25 \%$ of the isolates recovered from virulent cankers in the central Appalachians was not associated with hypovirulence or altered morphology (15). Similarly, no general correlations between the presence of dsRNA and virulence were reported for $R$. solani $(2,3,23)$, although associations between specific segments of dsRNA and hypovirulence were proposed (2). Associations between dsRNA and hypovirulence must be considered as a dsRNA-specific phenomenon. Recently, Choi and Nuss (12) reported that a full-length cDNA copy of a hypovirulence-associated dsRNA (CHV1-713) conferred the hypovirulence phenotype when introduced into virulent strains of $C$. parasitica by DNA-mediated transformation. The stable maintenance of integrated viral cDNA through repeated rounds of asexual sporulation and passages on host plant tissue also has been demonstrated (11).

The objectives of our study were to identify hypovirulent isolates of $S$. homoeocarpa, determine if hypovirulence was associated with specific dsRNA(s), and identify hypovirulent isolates with potential for future studies in biological control. A portion of these results has been published previously (37).

\section{MATERIALS AND METHODS}

Isolates. Isolates of $S$. homoeocarpa were obtained from the culture collections of G. J. Boland and T. Hsiang, University of Guelph, where they were maintained as pure cultures grown on cereal grains and stored at $-10^{\circ} \mathrm{C}$. The origins of the 132 isolates are listed in Table 1. Before further use, all isolates were subcultured onto potato dextrose agar (PDA, infusion of $200 \mathrm{~g}$ of potato, $20 \mathrm{~g}$ of dextrose, and $7.5 \mathrm{~g}$ of purified agar for 1 liter of medium).

Virulence evaluation. Virulence of $S$. homoeocarpa isolates was evaluated on creeping bentgrass (Agrostis palustris Hudson) in both laboratory and field experiments. For the field experiment, 
132 isolates were cultured in glass vials containing approximately $3 \mathrm{~cm}^{3}$ of sand:cornmeal medium $(2: 1 \mathrm{vol} / \mathrm{vol})$. The cultures were incubated at room temperature $\left(20\right.$ to $\left.22^{\circ} \mathrm{C}\right)$ for at least 2 weeks. Small aliquots of the colonized sand:cornmeal medium were placed in the middle of $5 \times 5-\mathrm{cm}$ squares on swards of creeping bentgrass at the Cambridge Research Station, Ontario, Canada. The experiment was conducted as a randomized complete block design with four replications. Ratings were made 2 and 4 weeks after inoculation for the presence or absence of symptoms of dollar spot.

For the laboratory experiments, detached leaves of creeping bentgrass (cv. Cobra) were used for evaluating virulence of selected isolates only (e.g., isolates containing dsRNA). Grass seed was sown in a pasteurized greenhouse soil mix in plastic pots (75 $\times 100 \mathrm{~mm}$ ), and leaves of the grasses were collected 4 weeks after sowing. The detached leaves were cut into segments 35-mm long, surface-disinfested with $0.5 \%$ sodium hypochlorite for $30 \mathrm{~s}$, and then rinsed three times with sterile water. Three segments of leaves were placed in a plastic petri dish $(100 \times 15 \mathrm{~mm})$ on top of a 90-mm-diameter filter paper (Whatman No. 1), where one end of each the three leaf segments touched together in the center of the petri dish and the other ends radiated away at $120^{\circ}$. The filter paper was moistened with $2.5 \mathrm{ml}$ of sterile water containing 30 $\mu \mathrm{g} / \mathrm{ml}$ of benzimidazole (Sigma Chemical Co., St. Louis). Isolates of $S$. homoeocarpa were cultured on PDA for 5 to 7 days. A 7mm-diameter plug of PDA, colonized by individual isolates to be tested, was placed onto the center of the petri dish where the ends of the three leaf segments touched together. Isolate Sh48 was selected as a typical, representative isolate of $S$. homoeocarpa, based on morphology and virulence on creeping bentgrass. Uncolonized plugs were used in control treatments. The petri dishes were sealed with Parafilm and incubated at room temperature (20 to $22^{\circ} \mathrm{C}$ ) under fluorescent lights (photoperiod of $12 \mathrm{~h}$ ). The petri dishes were arranged as a completely randomized design with three replications (plates) per isolate. Lesion length on each detached leaf was measured 3 and 5 days postinoculation. The experiment was repeated once.

Extraction and purification of dsRNA. All isolates evaluated for virulence also were tested for the presence of dsRNA. Isolates were cultured on PDA for 14 days at 20 to $22^{\circ} \mathrm{C}$. Mycelium ( $20 \mathrm{~g}$, PDA colonized by mycelium) was immersed in liquid nitrogen and ground to a fine powder using a mortar and pestle. The ground mycelium was suspended in extraction buffer $(2 \times$ Tris$\mathrm{HCl}, \mathrm{NaCl}$, and EDTA [STE]; $0.4 \mathrm{ml}$ of 2-mercaptoethanol; $10 \mathrm{ml}$ of $10 \%$ sodium dodecyl sulfate; and $30 \mathrm{ml}$ of phenol saturated with $2 \times$ STE), shaken at $200 \mathrm{rpm}$ for $30 \mathrm{~min}$ at room temperature, and centrifuged at $10,000 \times g$ for $20 \mathrm{~min}$. The aqueous phase was collected, adjusted to $16.5 \%$ ethanol, and poured onto a column prepared with $2.5 \mathrm{~g}$ of $\mathrm{CF}-11$ cellulose powder saturated with

TABLE 1. Source and origin of isolates of Sclerotinia homoeocarpa

\begin{tabular}{|c|c|c|c|}
\hline Isolate & Host & Location & No. \\
\hline $\begin{array}{l}\text { Sh01, ..., Sh45, Sh80, } \\
\text { Sh6aL, Sh6bL, Sh11L, } \\
\text { Sh12L, Sh16L, Sh16bL, } \\
\text { Sh6D, Sh06aD, Sh06bD, } \\
\text { Sh11D, Sh12D, Sh15D }\end{array}$ & Agrostis palustris & Cambridge, ON, Canada & 58 \\
\hline $\begin{array}{l}\text { Sh84, Sh14D, Sh14aD, } \\
\text { Sh14bD, Sh14L, Sh14aL, } \\
\text { Sh15L }\end{array}$ & Agrostis palustris & PA, United States & 7 \\
\hline $\begin{array}{l}\text { Sh2aL, Sh13L, Sh02D, } \\
\text { Sh02aD, Sh02bD, Sh13D }\end{array}$ & Роа аппиа & Cambridge, ON, Canada & 6 \\
\hline $\begin{array}{l}\text { Sh01D, Sh03D, ..., Sh05D, } \\
\text { Sh07D }\end{array}$ & Poa pratensis & Cambridge, ON, Canada & 5 \\
\hline $\begin{array}{l}\text { Sh08D, ..., Sh10D, Sh16D, } \\
\text { Sh18D, Sh01B, ..., Sh50B, } \\
\text { Sh286B }\end{array}$ & Festuca spp. & Cambridge, ON, Canada & 56 \\
\hline
\end{tabular}

STE-16.5\% ethanol (28). The column was washed with $80 \mathrm{ml}$ STE-16.5\% ethanol and purged with air. Fifteen milliliters of STE in three aliquots was used to elute any dsRNA bound to the CF-11 cellulose. RNase $\mathrm{T}_{1}(2.5 \mu \mathrm{g} / \mathrm{ml}$; Boehringer Mannheim Biochemicals, Laval, Quebec, Canada) was added, and the sample was digested at $37^{\circ} \mathrm{C}$ for $30 \mathrm{~min}$. One molar $\mathrm{MgCl}_{2}(0.5 \mathrm{ml})$ was added to the sample, and DNase I (1 unit $/ \mathrm{ml}$; Boehringer Mannheim Biochemicals) digestion was performed at $37^{\circ} \mathrm{C}$ for $30 \mathrm{~min}$. Next, $0.3 \mathrm{~g}$ of cellulose (Cellex N-1; Bio-Rad Laboratories, Mississauga, Ontario, Canada) was added, and the mixture was shaken at room temperature for $20 \mathrm{~min}$. The sample was poured onto a second column and washed with $20 \mathrm{ml}$ of STE-16.5\% EtOH. The column was purged and then eluted with $3 \mathrm{ml}$ of STE. The sample was centrifuged at $6,000 \times g$ for $1 \mathrm{~min}$. Three $\mathrm{M}$ Na acetate $(0.1 \mathrm{vol}-$ ume) and $95 \%$ ethanol ( 2.5 volumes) were added to the tube, and the sample was precipitated overnight at $-20^{\circ} \mathrm{C}$. Precipitates were collected by centrifugation at $8,000 \times g$ for $10 \mathrm{~min}$, and pellets were dissolved in $30 \mu \mathrm{l}$ of sterile distilled water. Samples were electrophoresed through $0.8 \%$ agarose gels with ethidium bromide $(0.75 \mu \mathrm{g} / \mathrm{ml})$ in electrophoresis buffer for 2 to $3 \mathrm{~h}$ at 25 to $30 \mathrm{~mA}$. Gels were destained in distilled water for $15 \mathrm{~min}$ and then photographed with Polaroid Type 665 film (Polaroid Corp., Cambridge, MA) using a $23 \mathrm{~A}$ red filter and ultraviolet light source $(302 \mathrm{~nm})$. Isolates in which dsRNA was detected were denoted as dsRNA positive $\left(\mathrm{dsRNA}^{+}\right)$isolates.

Confirmation of dsRNA. Enzymatic digestions of nucleic acid samples were conducted with deoxyribonuclease (DNase I; Sigma Chemical Co.) in $0.01 \mathrm{M} \mathrm{MgCl}_{2}$ at 10 units $/ \mathrm{ml}$ at $37^{\circ} \mathrm{C}$ for 60 min, and pancreatic RNase (Sigma Chemical Co.) in $0.03 \mathrm{M}$ or $0.3 \mathrm{M} \mathrm{NaCl}$ solution containing $2.5 \mu \mathrm{g} / \mathrm{ml}$ at $37^{\circ} \mathrm{C}$ for $60 \mathrm{~min}$. Nucleic acids that hydrolyzed in the $0.03 \mathrm{M}$ RNase treatment, but not the $0.3 \mathrm{M}$ treatment or the DNase treatment, were considered to be dsRNA (1).

Transmission of hypovirulence and dsRNA. Two methods were used to determine if hypovirulence and dsRNA were transmissible through hyphal anastomosis. One of the methods was modified from Boland (4). A colonized agar plug of the hypovirulent dsRNA ${ }^{+}$isolate Sh12B of $S$. homoeocarpa was placed in the center of a PDA plate. After 4 to 7 days, two to three plugs were cut from the actively growing margin of a virulent dsRNA negative (dsRNA $\left.{ }^{-}\right)$isolate and transferred onto the outer margin of the established hypovirulent colony. Hyphal tips were then isolated from the virulent colonies, which grew away from the hypovirulent colony 3 to 5 days later. The slower growth of the hypovirulent isolate, in comparison with that of the virulent isolates, permitted a clear identification of the two colonies when subculturing from mycelium of the recipient isolate. In the second method, a tebuconazole-resistant isolate of S. homoeocarpa (KY7) from creeping bentgrass in Kentucky (provided by J. C. Doney, Jr., University of Kentucky, Lexington) was used to confirm transmission of dsRNA from donor to recipient isolates. This isolate was evaluated twice to ensure that it was dsRNA ${ }^{-}$. To ensure the fungicide resistance of dsRNA ${ }^{-}$isolate $\mathrm{KY}-7$ and the sensitivity of dsRNA ${ }^{+}$isolate Sh12B, both isolates were cultured on PDA and PDA amended with $2 \mu \mathrm{g}$ a.i./ml of tebuconazole (BayHWG 1608; Bayer AG, Leverkusen, Germany) at 12 plates/medium/ isolate. Colony diameter of the cultures was measured every week for 10 weeks. To transfer dsRNA from isolate Sh12B to tebuconazole-resistant isolate KY-7, colonized agar plugs of the two isolates were placed side by side on a PDA plate and incubated at room temperature until the hyphae of the two isolates made contact. Agar plugs were then removed from the contact area on the plate and subcultured on PDA amended with $2 \mu \mathrm{g}$ a.i./ml of tebuconazole. As controls, agar plugs from isolates Sh12B and KY-7 also were transferred onto PDA amended with tebuconazole. All subcultures from both methods were transferred to a fresh PDA plate for comparisons of morphology, virulence, and presence of dsRNA as described previously. 
Interactions of $\mathrm{dsRNA}^{+}$and $\mathrm{dsRNA}^{-}$isolates in plant tissues. Two tests were conducted on apple fruits and detached leaves of creeping bentgrass to determine if dsRNA transfer could occur in plant tissues. Apples (cv. Granny Smith) were surfacedisinfested with $0.5 \%$ sodium hypochlorite for $2 \mathrm{~min}$ and rinsed three times with sterile water. A 7-mm-diameter $\times 5$-mm-deep plug of fruit tissue was removed with a sterile cork borer for inoculation. Two fungal colonized agar plugs, one from a hypovirulent and the other from a virulent isolate, were placed in the apple tissues with mycelial sides facing each other and then covered with cellophane tape. Treatments were randomly assigned to individual apples, and each apple was inoculated at four locations. Each treatment was replicated three times. The inoculated apples were arranged in a completely randomized design in a plastic crisper placed at $20^{\circ} \mathrm{C}$ in the dark. After 10 to 15 days, lesion diameters on the apple were measured, and $S$. homoeocarpa was reisolated from rotted tissues. Detached leaves of creeping bentgrass were treated and arranged in a petri dish as described in the virulence test. Two agar plugs were placed on the ends of the leaf pieces that touched together in the center, in the same manner as the inoculation of apple fruits. Three petri dishes were used for each treatment, and these were incubated at the same conditions described previously. Length of lesions on the leaves were recorded 3 and 4 days after inoculation. To reisolate S. homoeocarpa, the infected leaf tissues were surface-disinfested with $0.5 \%$ sodium hypochlorite for $30 \mathrm{~s}$, rinsed with sterile water, placed on PDA, and allowed to grow for 2 to 4 weeks. Resulting colonies were subcultured and evaluated for the presence of dsRNA.

Curing of dsRNA ${ }^{+}$isolates. The influence of dsRNA on selected isolates was examined by treatment with cycloheximide, heat, or both to recover isolates that were dsRNA ${ }^{-}(17,19)$. Isolates were first cultured on PDA for 5 to 7 days, and then plugs from the actively growing margin were transferred onto PDA amended with cycloheximide (Sigma Chemical Co.) at 0, 10, 15, and $20 \mu \mathrm{g} / \mathrm{ml}$. Plates containing the same concentration of cycloheximide were separated into four groups, and each group was placed in one of four incubators at $20,25,28$, and $30^{\circ} \mathrm{C}$. There were five plates for each combination of cycloheximide concentration and temperature. After 14 days, hyphal tips were taken from rapidly growing sectors of individual colonies and subcul- tured on fresh PDA plates at 20 to $22^{\circ} \mathrm{C}$. Resulting colonies were evaluated for colony morphology, virulence, and the presence of dsRNA.

Selection of dsRNA-free cells. Attempts to obtain dsRNA-free cells from dsRNA ${ }^{+}$isolates were made through selection of protoplasts. Cultures of isolates Sh12B and Sh48B were grown in 50 $\mathrm{ml}$ of potato dextrose broth (infusion of $200 \mathrm{~g}$ of potato and $20 \mathrm{~g}$ of dextrose for 1 liter of medium) on a rotary shaker operated at $100 \mathrm{rpm}$ at 22 to $25^{\circ} \mathrm{C}$ for 5 to 7 days. Protoplasts prepared from vegetative hyphae (35) were diluted to $10^{6}$ cells $/ \mathrm{ml}$ with STC buffer (1.2 M sorbitol, $10 \mathrm{mM}$ Tris- $\mathrm{HCl}$ [pH 7.5], and $10 \mathrm{mM}$ $\left.\mathrm{CaCl}_{2}\right)$. Aliquots $(100 \mu \mathrm{l})$ were spread on regeneration medium (1.2 M sorbitol and $1.5 \%$ agar) in a $15 \times 100-\mathrm{mm}$ plastic petri dish (10 dishes/isolate). After 3 to 5 days at room temperature, fastgrowing, single, regenerated protoplasts, 20 to 80 per isolate, were identified using a dissecting microscope and transferred onto PDA plates (four protoplasts/plate). Each of the resulting colonies was subcultured once for evaluation of cultural morphology and virulence. Colonies that were similar in morphology to typical isolates were tested for virulence and the presence of dsRNA.

\section{RESULTS}

Morphology of isolates. Most isolates in this study displayed the typical characteristics of $S$. homoeocarpa in culture (16). White mycelia covered the PDA plates in 5 to 7 days when grown at room temperature, and stroma formed after an additional 1 to 2 weeks. Mature colonies were characterized by the black paperthin stroma that developed over most of the culture (Fig. 1). However, isolates Sh08D, Sh09B, Sh13B, and Sh12B grew slowly on PDA and formed thin colonies with atypical margins. Mature colonies were brown to dark brown, lacked stromata, and had a mycelial strandlike appearance (Fig. 1).

Hypovirulence and presence of dsRNA. One hundred and thirty-two isolates of $S$. homoeocarpa (Table 1) were evaluated for virulence on swards of creeping bentgrass. Among these isolates, $24(18.2 \%)$ did not cause any symptoms of dollar spot 2 weeks after inoculation. After an additional 2 weeks, there were 13 isolates $(9.8 \%)$ that still had not initiated lesions in any of the four inoculated sites. All of these isolates were considered to be hy-

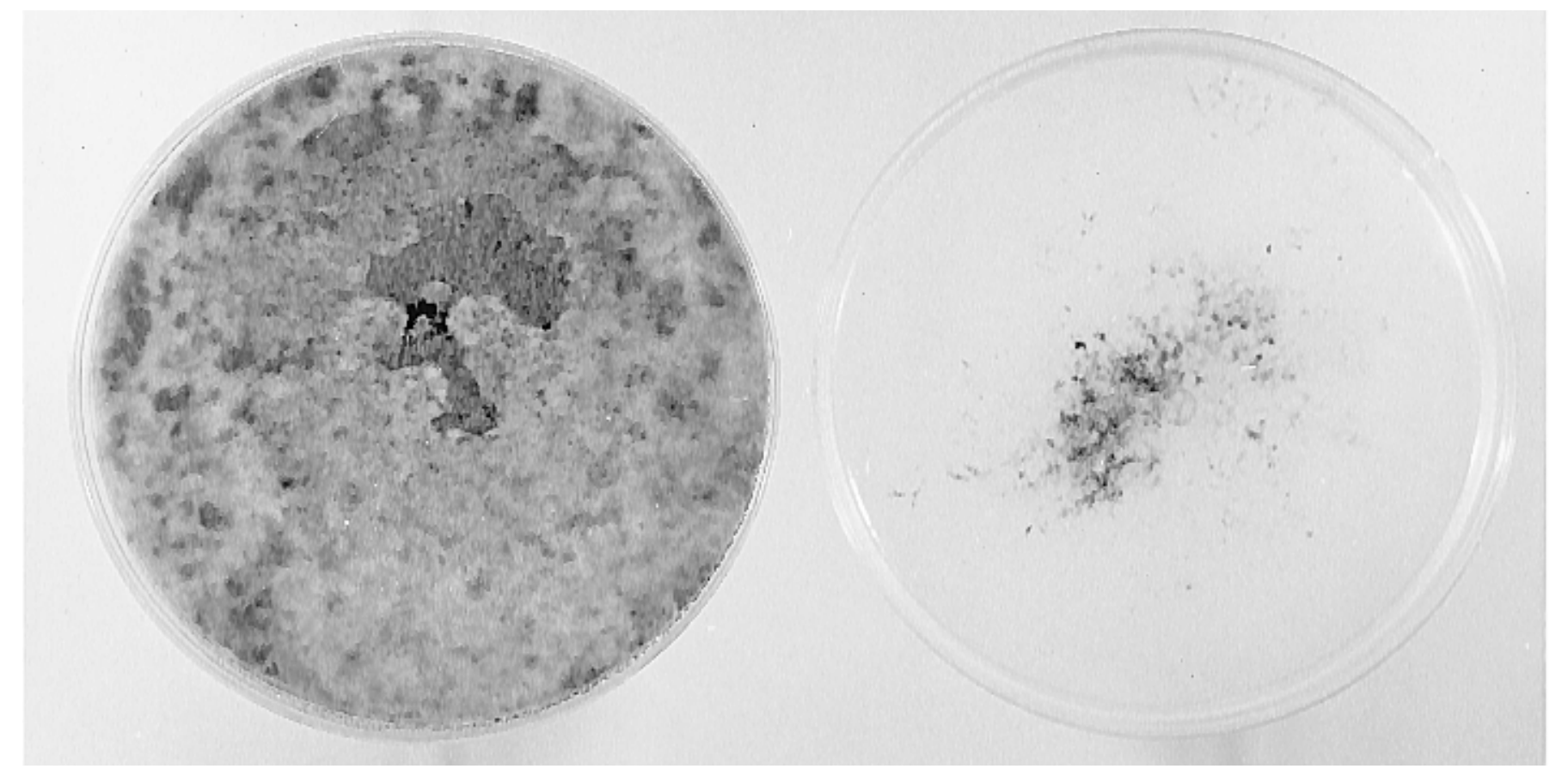

Fig. 1. Comparative morphology of hypovirulent dsRNA+ isolate Sh12B of Sclerotinia homoeocarpa (right) and normal virulent isolate Sh48B (left) after 2 weeks of growth on potato dextrose agar medium at $20^{\circ} \mathrm{C}$. 
povirulent, because they had a greatly reduced ability to initiate disease in comparison with other isolates.

All 132 isolates evaluated in the field were tested for the presence of dsRNA. Nucleic acids from different isolates were subjected to agarose gel electrophoresis. One to three segments (bands) were visible from various isolates of $S$. homoeocarpa (Fig. 2). These segments were resistant to digestion by DNase and resistant to RNase at high ionic strength $(0.3 \mathrm{M} \mathrm{NaCl})$, but sensitive to RNase at low ionic strength (0.03 M NaCl) (Fig. 3) and, therefore, were considered to be dsRNA (1). The size of dsRNA segments obtained from different isolates varied, although there was one segment $(2.7 \mathrm{~kb})$ in common to all dsRNA ${ }^{+}$isolates (Fig. 2). In this study, an isolate was considered to be dsRNA ${ }^{+}$if at least one dsRNA segment was detected on the agarose gel. dsRNA was detected in 15 of the 132 isolates $(11.4 \%)$ that were assessed. In nine of these isolates, the presence of dsRNA did not appear to be associated with hypovirulence. However, in isolates Sh08D, Sh09B, Sh12B, Sh13B, Sh42B, and Sh46B, the presence of dsRNA was associated with an absence of disease symptoms in the 2- or 4-week evaluations (Table 2).

All 15 isolates that were dsRNA ${ }^{+}$in the first test (Table 2), except isolate Sh46B, also were evaluated for virulence using detached bentgrass leaves. In the first detached-leaf test, isolates Sh02D, Sh06B, Sh08D, Sh09B, Sh2aL, Sh12B, Sh18D, and Sh42B caused smaller lesions compared with the typical virulent isolate Sh48B (Table 2). In the second test, grass leaves inoculated with isolates Sh2aL, Sh30B, Sh42B, Sh43B, and Sh44B developed lesions that were similar in length to those caused by isolate Sh48B, however, grass leaves inoculated with all remaining isolates developed smaller lesions than those caused by isolate Sh48B (Table 2). Subcultures from these isolates were evaluated twice for the presence of dsRNA. Of 14 subcultures that were tested, dsRNA was detected in isolates Sh09B, Sh12B, Sh13B, Sh18D, and Sh30B in both tests and in isolate Sh08D only in the first test (Table 2). dsRNA was not detected in the other isolates.

Transmission of dsRNA and hypovirulence. Hyphal tips subcultured from colonies of virulent isolates Sh48B, Sh285B, Sh13D, and Sh14D that had grown away from the hypovirulent colony of Sh12B grew very slowly after they were transferred to fresh PDA plates. Colony diameters of these subcultures were only 5 to $20 \mathrm{~mm}$ after incubation for 3 to 6 weeks at room temperature compared with virulent isolates, which covered the plates within 1 week, and hypovirulent isolates, which covered the plates in 2 weeks (data not shown). However, the growth of some subcultures increased unexpectedly after this period of 3 to 6 weeks, and mycelium subsequently covered plates within 7 to 10 days. These subcultures were evaluated for virulence on detached creeping bentgrass leaves. Lesion lengths caused by these sub-

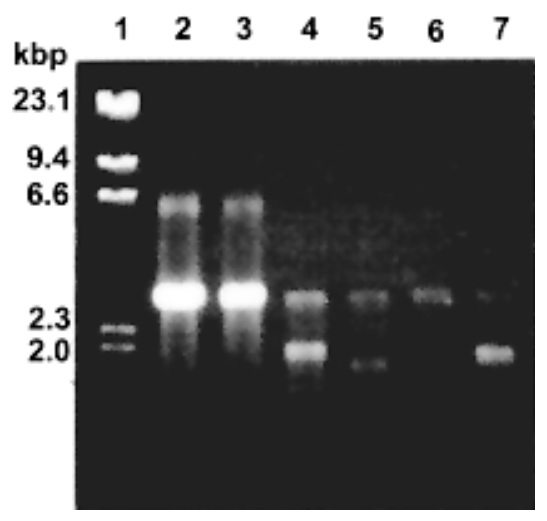

Fig. 2. dsRNA segment patterns on agarose electrophoresis gel $(0.8 \%)$ stained with ethidium bromide. Lane $1, \lambda$ DNA cut with HindIII as molecular weight markers. Lanes 2 to 6, dsRNA from isolates Sh12B, Sh08D, Sh09B, Sh13B, Sh18D, and Sh30B of Sclerotinia homoeocarpa, respectively. cultures ranged from 0 to $8.5 \mathrm{~mm}$ after 3 days of incubation and were significantly smaller than lesions initiated by their parent isolates (24.3 to $32.0 \mathrm{~mm}$ ), but were similar to the hypovirulent parent isolate Sh12B (5.2 mm). Morphologically, subcultures from the hyphal tips were similar to isolate Sh12B, but not to the parent isolates. dsRNA was detected in all subcultures resulting from the hyphal tip isolations.

Similar results were found when the fungicide-resistant, dsRNA ${ }^{-}$isolate KY-7 was paired with Sh12B. Agar plugs removed from the contact area of isolates KY-7 and Sh12B formed colonies on PDA amended with tebuconazole $(2 \mu \mathrm{g} / \mathrm{ml})$ after 2 weeks. Colonies of the fungicide-resistant isolate KY-7 and fungicide-sensitive isolate Sh12B averaged 8.5 and $0 \mathrm{~cm}$, respectively, after 2 weeks of growth on fungicide-amended PDA. The fungicide-sensitive isolate Sh12B did not grow on fungicide-amended medium for up to 10 weeks of incubation. Isolates subcultured from the contact area of isolates KY-7 and Sh12B were able to grow on fungicide-amended medium, but at a reduced rate compared with isolate KY-7. This reduced rate of growth, as also observed in the previous method, was associated with the inconsistent growth of many subcultures during the experimental period. Most of these subcultures initially did not grow for 2 to 6 weeks, but then unexpectedly started growing and subsequently developed colonies typical of the hypovirulent isolate on the fungicideamended medium. Colony diameters of isolates subcultured from the contact area of isolates KY-7 and Sh12B and grown on fungicide-amended medium for 2 weeks ranged from 0 to $8.5 \mathrm{~cm}$ and averaged $2.2 \mathrm{~cm}$. After 10 weeks of growth on fungicide-amended medium, colony diameters of the KY-7 $\times$ Sh12B isolates averaged $7.9 \mathrm{~cm}$ compared with the fungicide-sensitive isolate Sh12B, which had an average colony diameter of $0 \mathrm{~cm}$. Therefore, these KY-7 $\times$ Sh12B colonies were considered to be subcultures of KY7 , because only KY-7 can grow on the fungicide-amended medium. Thirteen subcultures had similar colony morphologies to Sh12B and were dsRNA ${ }^{+}$.

Interactions of $\mathbf{d s R N A}^{+}$and dsRNA ${ }^{-}$isolates. When apple fruits and detached leaves of creeping bentgrass were inoculated with isolate Sh48B together with dsRNA ${ }^{+}$isolate Sh12B, the size of lesions on both apple fruits and detached creeping bentgrass were intermediate as compared with treatment with only isolate Sh48B (44.2 to $50.3 \%$ reduction) and treatment with only Sh12B (Table 3). Isolates recovered from diseased apple fruit and bentgrass leaves from treatments of Sh48B plus Sh12B and Sh12B only were hypovirulent and dsRNA ${ }^{+}$(Table 3 ).

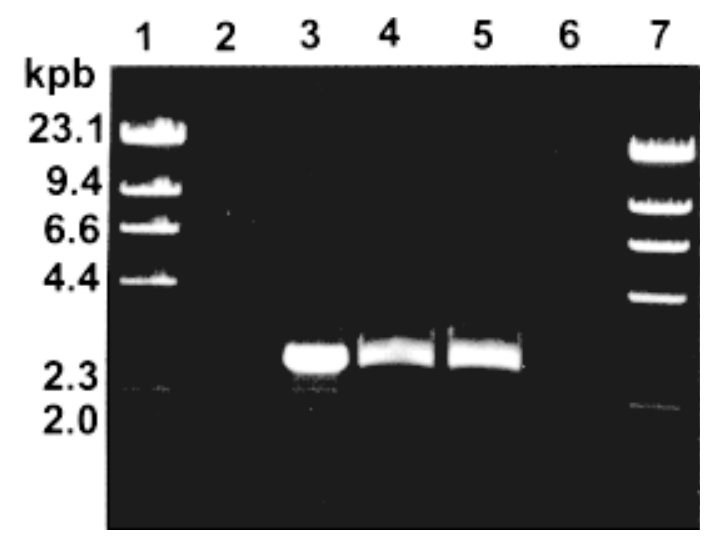

Fig. 3. DNase I and pancreatic RNase treatment of dsRNA extracted from isolate Sh12B of Sclerotinia homoeocarpa on agarose electrophoresis gel $(0.8 \%)$ stained with ethidium bromide. Lanes 1 and $7, \lambda$ DNA cut with HindIII as molecular weight markers. Lane $2, \lambda$ DNA digested with DNase I in $0.01 \mathrm{M} \mathrm{MgCl}_{2}$ as a control treatment. Lane 3, untreated dsRNA from isolate Sh12B. Lane 4, dsRNA from isolate Sh12B digested with DNase I. Lane 5, dsRNA from isolate Sh12B digested with pancreatic RNase in $0.3 \mathrm{M} \mathrm{NaCl}$. Lane 6, dsRNA from isolate Sh12B digested with pancreatic RNase in 0.03 $\mathrm{M} \mathrm{NaCl}$. 
Curing of dsRNA ${ }^{+}$isolates. Cured subcultures were obtained from isolate $\mathrm{Sh} 12 \mathrm{~B}$ grown on a medium containing $20 \mu \mathrm{g}$ of cycloheximide $/ \mathrm{ml}$ at $28^{\circ} \mathrm{C}$ and on a medium with 10,15 , or $20 \mu \mathrm{g}$ of cycloheximide $/ \mathrm{ml}$ at $30^{\circ} \mathrm{C}$. These hyphal tip subcultures grew faster than untreated cultures of Sh12B and had colony morphologies that were similar to typical virulent isolates of $S$. homoeocarpa. These typical cultural characters were still evident after three serial subcultures. In the virulence test on detached leaves of creeping bentgrass, lesion lengths resulting from these cured subcultures were 20 to $23 \mathrm{~mm}$ measured 3 days after inoculation and were similar to $23 \mathrm{~mm}$ from the virulent isolate Sh48B, but significantly different from $2 \mathrm{~mm}$, caused by isolate Sh12B (Table 4). dsRNA was not detected from these hyphal tip subcultures. No cured subcultures were obtained from isolate Sh09B, although hyphal tip subcultures from colonies treated with a variety of temperature and cycloheximide treatments varied in morphology and growth rate from the untreated culture. For all tested isolates, Sh09B, Sh12B, and Sh48B, higher temperatures $\left(28\right.$ and $\left.30^{\circ} \mathrm{C}\right)$ and concentrations of cycloheximide in the medium (10 to 20 $\mu \mathrm{g} / \mathrm{ml}$ ) inhibited mycelial growth.

Selection of dsRNA-free cells. Isolates regenerated from the relatively fast-growing, single protoplasts of isolate Sh12B had considerable variation in morphology. Of 80 regenerated isolates, three isolates were similar to virulent dsRNA ${ }^{-}$isolate Sh48B in morphology and growth rate. However, although these isolates were more virulent than isolate Sh12B, none of these isolates were as virulent as $\mathrm{Sh} 48 \mathrm{~B}$ when tested on detached leaves of creeping bentgrass (data not shown). dsRNA was detected from all regenerated cultures of Sh12B. All subcultures derived from regenerated protoplasts of isolate $\mathrm{Sh} 48 \mathrm{~B}$ displayed colony morphologies typical of the parent isolate.

\section{DISCUSSION}

Although hypovirulence and the presence of dsRNA have been reported for a number of plant pathogenic fungi, this is the first report of hypovirulence and dsRNA in $S$. homoeocarpa and a possible association between these two factors. Hypovirulence has been reported from numerous plant pathogenic fungi and, in many cases, associated with the presence of mycoviruses or unencapsi- dated dsRNA $(4,8,9,14,19,27,30,36)$; some of the latter have been classified within the unique virus family Hypoviridae (20). dsRNA-associated hypovirulence in plant pathogenic fungi has attracted considerable interest in recent years, because of the potential of these novel host-parasite interactions to provide insight into the regulation of virulence in plant pathogens and also because of their potential for biological control of diseases. The discovery of transmissible hypovirulence in S. homoeocarpa that is associated with dsRNA will enable such studies for this economically important plant pathogen.

The virulence of isolates of $S$. homoeocarpa was evaluated on swards and detached leaves of creeping bentgrass. Symptoms of dollar spot are unique compared with many other diseases. After initiation, lesions expand for a relatively short while, but then stop expanding, typically at the size of "dollar spots." Therefore, the number of spots that developed in experimental plots was used to measure virulence in field conditions. Isolates that did not initiate symptoms of dollar spot at all inoculation sites were considered to be potential hypovirulent isolates. Selected isolates were then evaluated using a detached creeping bentgrass leaf assay to provide a more quantitative measure of virulence based on lesion length. Curiously, lesion expansion in the detached leaf assay was continuous and did not reach a maximum size within the experimental period. With few exceptions, the results on detached leaves agreed with those on swards.

Several patterns of dsRNA were observed among isolates that contained detectable concentrations of dsRNA. The presence of dsRNA in these isolates suggests the presence of mycoviruses, virus-like particles, hypoviruses, or virus-like nucleic acids $(7,8$, $20,30)$. The varying patterns of dsRNA observed in these isolates following agarose electrophoresis may or may not represent unique genetic elements. Hybridization analyses are required to verify the genetic relatedness among these dsRNA segments. The status of dsRNA in cells of $S$. homoeocarpa was not examined, but attempts to isolate and purify virus or virus-like particles from isolate Sh12B using two methods $(6,25)$ were unsuccessful. Similar results were reported for attempts to characterize the source of dsRNA in $S$. sclerotiorum (5). Mycovirus particles were not detected in a dsRNA-containing isolate of this fungus, but dsRNA was associated with double membrane bodies within mycelia and

TABLE 2. Association between the presence of dsRNA and virulence in isolates of Sclerotinia homoeocarpa

\begin{tabular}{|c|c|c|c|c|c|c|c|}
\hline \multirow[b]{3}{*}{ Isolate $^{w}$} & \multicolumn{3}{|c|}{ On swards ${ }^{\mathrm{x}}$} & \multicolumn{2}{|c|}{ On leaf (test 1$)^{\mathrm{y}}$} & \multicolumn{2}{|c|}{ On leaf (test 2) } \\
\hline & & \multicolumn{2}{|c|}{ No. of sites diseased at } & \multirow[b]{2}{*}{ dsRNA $^{z}$} & \multirow[b]{2}{*}{ Length of lesion (mm) } & \multirow[b]{2}{*}{$\mathrm{dsRNA}^{\mathrm{z}}$} & \multirow[b]{2}{*}{ Length of lesion (mm) } \\
\hline & dsRNA $^{z}$ & 2 weeks & 4 weeks & & & & \\
\hline Sh48B & - & 4 & 4 & - & 23 & - & 34 \\
\hline Sh06B & + & 3 & 4 & - & $13^{*}$ & - & $24 *$ \\
\hline Sh18D & + & 3 & 4 & + & $11 *$ & + & $14^{*}$ \\
\hline Sh44B & + & 2 & 4 & - & 25 & - & 34 \\
\hline Sh31B & + & 2 & 4 & - & 17 & - & $25^{*}$ \\
\hline Sh43B & + & 2 & 4 & - & 16 & - & 34 \\
\hline Sh04B & + & 1 & 4 & - & 16 & - & $23^{*}$ \\
\hline Sh02D & + & 1 & 4 & - & $10 *$ & - & $24 *$ \\
\hline Sh08D & + & 0 & 4 & + & $0^{*}$ & - & $21 *$ \\
\hline $\mathrm{Sh} 2 \mathrm{aL}$ & + & 3 & 3 & - & $14^{*}$ & - & $26^{*}$ \\
\hline Sh30B & + & 1 & 3 & + & 17 & + & 29 \\
\hline Sh46B & + & 0 & 2 & $\sim$ & $\sim$ & $\sim$ & $\sim$ \\
\hline Sh13B & + & 0 & 0 & + & 16 & + & $7 *$ \\
\hline Sh42B & + & 0 & 0 & - & $13^{*}$ & - & 33 \\
\hline Sh09B & + & 1 & 0 & + & $0^{*}$ & + & $18^{*}$ \\
\hline Sh12B & + & 1 & 0 & + & $0^{*}$ & + & $0^{*}$ \\
\hline Control & & 0 & 0 & & 0 & & 0 \\
\hline
\end{tabular}

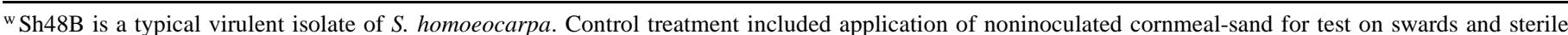
agar plug for test on detached leaves.

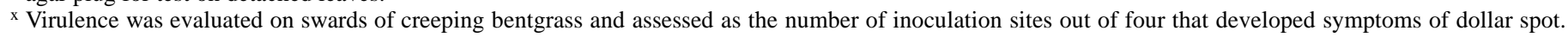
Assessments were made at 2 and 4 weeks after inoculation, and the results are shown in the second and the third column, respectively.

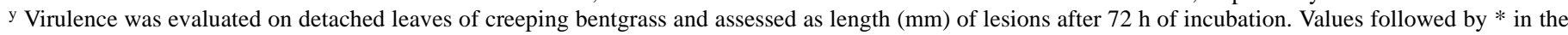
same column are significantly different from virulent control isolate Sh48B according to Dunnett's $t$ test $(P=0.05)$.

${ }^{\mathrm{z}}$ Cultures grown on potato dextrose agar were evaluated for presence $(+)$ or absence $(-)$ of dsRNA. 
TABLE 3. Interactions of dsRNA ${ }^{+}$and dsRNA ${ }^{-}$isolates of Sclerotinia homoeocarpa on plant tissues

\begin{tabular}{|c|c|c|c|c|c|c|}
\hline \multirow[b]{3}{*}{ Isolates $^{\mathrm{v}}$} & \multicolumn{3}{|c|}{ Apple fruits } & \multicolumn{3}{|c|}{ Detached leaves } \\
\hline & \multicolumn{2}{|c|}{ Lesion diameter $(\mathrm{mm})^{\mathrm{w}}$} & & \multicolumn{2}{|c|}{ Lesion length $(\mathrm{mm})^{\mathrm{x}}$} & \\
\hline & Original $^{\mathrm{y}}$ & Reisolate $^{y}$ & $\mathrm{dsRNA}^{\mathrm{z}}$ & Original $^{\mathrm{y}}$ & Reisolate $^{\mathrm{y}}$ & dsRNA $^{z}$ \\
\hline Sh $48 B$ + PDA & $28 \mathrm{a}$ & & - & $27 \mathrm{a}$ & & - \\
\hline Sh48B & $28 \mathrm{a}$ & $32 \mathrm{a}$ & - & $30 \mathrm{a}$ & $35 \mathrm{a}$ & - \\
\hline Sh $48 B+\operatorname{Sh} 12 B$ & $14 \mathrm{~b}$ & $3 \mathrm{~b}$ & + & $15 \mathrm{~b}$ & $1 \mathrm{~b}$ & + \\
\hline Sh12B + PDA & $5 \mathrm{c}$ & $2 \mathrm{~b}$ & + & $0 \mathrm{c}$ & $\ldots$ & + \\
\hline PDA & 0 & $\ldots$ & & $0 \mathrm{c}$ & $\ldots$ & \\
\hline
\end{tabular}

${ }^{v}$ Sh48B was a typical virulent dsRNA ${ }^{-}$isolate. Sh12B was a hypovirulent dsRNA $^{+}$isolate. PDA indicates sterile potato dextrose agar plugs. As two isolates were involved in a treatment, two plugs were coinoculated with one on top of the other with mycelial sides facing each other.

${ }^{w}$ Lesion diameter was measured 10 days after inoculation. Values are means of six replications (apples) pooled from two tests.

$x$ Virulence was tested on detached leaves of creeping bentgrass. Length of lesions was measured 3 days after inoculation. Values are means of three replications (plates).

${ }^{y}$ Means followed by the same letter in the column are not significantly different $(P=0.05)$ according to Dunnett's $t$ test.

${ }^{z}$ Presence of dsRNA was detected from PDA cultures of fungi reisolated from infected tissues.

sclerotia of the hypovirulent isolate only. Similarly, hypoviruses reported from $C$. parasitica have dsRNA genomes that are encapsulated in host-derived lipid vesicles $(13,20)$. Further study is needed to determine the status of dsRNA in $S$. homoeocarpa.

Hypovirulence was associated with dsRNA in selected isolates of S. homoeocarpa. Isolates Sh08D, Sh09B, Sh12B, and Sh13B displayed a markedly reduced ability to initiate disease in comparison with other isolates in swards and on detached leaves and contained dsRNA. These isolates were considered to be hypovirulent in association with dsRNA. Several additional isolates also may have displayed dsRNA-associated hypovirulence, but the association was variable. Other isolates had hypovirulent phenotypes that were not associated with detectable concentrations of dsRNA.

In isolate $\mathrm{Sh} 12 \mathrm{~B}$, the hypovirulent phenotype and the presence of dsRNA was consistent regardless of the number of serial transfers or type of culture media. Therefore, this isolate was selected for more extensive studies on curing and transmission of hypovirulence. Isolates cured of dsRNA using cycloheximide- and heat-regained virulence developed a typical culture morphology and did not contain detectable concentrations of dsRNA. In addition, the hypovirulent phenotype and dsRNA were transmitted from isolate $\mathrm{Sh} 12 \mathrm{~B}$ to recipient virulent isolates, and these recipient isolates developed the hypovirulent phenotype and contained dsRNA. Transmission of dsRNA between isolates was verified through the use of the fungicide-resistant isolate $\mathrm{Ky}-7$ as a recipient isolate. After pairing with isolate Sh12B, isolates derived from the recipient isolates contained dsRNA and grew on fungicide-amended medium. Also, hyphal anastomosis was observed between selected donor and recipient isolates of $S$. homoeocarpa using transmitted light microscopy. These results suggest that the hypovirulent phenotype in isolate $\mathrm{Sh} 12 \mathrm{~B}$ is associated with a unique banding pattern of dsRNA. However, hybridization and sequencing analyses are required to determine if the dsRNA segments associated with isolate Sh12B are unique.

dsRNA was detected in some isolates that were similar in virulence to typical dsRNA ${ }^{-}$isolates of $S$. homoeocarpa. This phenomenon has been reported for other pathogenic fungi. In $C$. parasitica, a 12-kb segment of dsRNA present in a relatively large proportion of virulent isolates was not associated with hypovirulence or altered morphology (15). Similarly, no general correlations between the presence of dsRNA and virulence were reported for $R$. solani $(2,3,23)$, although associations between specific segments of dsRNA and hypovirulence were proposed (2). Associations between dsRNA and hypovirulence must be considered a
dsRNA-specific phenomenon. Also, different patterns of dsRNA from different isolates and the inconsistent presence of dsRNA may partially explain the variation of virulence of dsRNA ${ }^{+}$isolates in S. homoeocarpa. Considerable variation in cross-hybridization among dsRNA has been reported from several fungi, suggesting that dsRNA in fungi are heterogeneous in their structure and influence on host isolates. Some are considered benign, whereas others are associated with hypovirulence. In addition, virus-resistant mutants have been found in $C$. parasitica (32).

The inconsistent growth of many subcultures following the transmission of hypovirulence and dsRNA from isolate Sh12B to virulent recipient isolates is similar to the "stop-start" growth pattern reported by Howitt et al. (22). In Botrytis cinerea, this growth pattern did not appear to be associated with the presence of dsRNA. In our study, only potentially converted isolates were examined, so no conclusions can be made on the association between this phenomenon and the presence of dsRNA. Because of this phenomenon, it was important to allow agar plugs removed from the contact area to incubate for extended periods of time to recover potentially converted isolates for confirmation of transmission.

Coinfecting detached leaves of creeping bentgrass and apple fruits with virulent isolate Sh48B and hypovirulent isolate Sh12B resulted in intermediate lesion lengths as compared with inoculation with individual isolates. Because the hypovirulent phenotype and dsRNA were successfully transmitted from isolate Sh12B to several virulent isolates in vitro, it seems likely that reductions of lesion size also were due to transmission of hypovirulent phenotype and dsRNA from Sh12B to virulent isolate Sh48B in vivo, although other mechanisms might be involved.

Transmission of dsRNA and associated hypovirulence in $S$. homoeocarpa has potential application as a novel approach to the management of dollar spot on turfgrass. The use of hypovirulent isolates to suppress dollar spot disease should be further evaluated under greenhouse and field conditions. Vegetative compatibility in $S$. homoeocarpa, the role of dsRNA in the determination of hypovirulence and debilitation, and the molecular ecology of dsRNA in the fungal population also should be addressed in future studies.

\section{ACKNOWLEDGMENTS}

We thank M. Wang, E. A. Smith, R. De Young, and D. Hamilton for technical assistance; and the Natural Sciences and Engineering Research Council of Canada and the Ontario Ministry of Agriculture, Food, and Rural Affairs for financial assistance.

\section{LITERATURE CITED}

1. Bellamy, A. R., Shapiro, L., August, J. T., and Joklik, W. K. 1967. Studies on reovirus RNA. I. Characterization of reovirus genome RNA. J. Mol. Biol. 29:1-17.

2. Bharathan, N., and Tavantzis, S. M. 1990. Genetic diversity of doublestranded RNA from Rhizoctonia solani. Phytopathology 80:631-635.

3. Bharathan, N., and Tavantzis, S. M. 1991. Assessment of genetic relatedness among double-stranded RNAs from isolates of Rhizoctonia solani from diverse geographic origins. Phytopathology 81:411-415.

4. Boland, G. J. 1992. Hypovirulence and double-stranded RNA in Sclerotinia sclerotiorum. Can. J. Plant Pathol. 14:10-17.

5. Boland, G. J., Mould, M. J. R., and Robb, J. 1993. Ultrastructure of a hypovirulent isolate of Sclerotinia sclerotiorum containing doublestranded RNA. Physiol. Mol. Plant Pathol. 43:21-32.

6. Bottacin, A. M., Lévesque, C. A., and Punja, Z. K. 1994. Characterization of dsRNA in Chalara elegans and effects on growth and virulence. Phytopathology 84:303-312.

7. Buck, K. W. 1986. Fungal virology-An overview. Pages 1-84 in: Fungal Virology. K. W. Buck, ed. CRC Press, Boca Raton, FL

8. Buck, K. W. 1987. Viruses of plant pathogenic fungi. Pages 111-126 in: Genetics and Plant Pathogenesis. P. R. Day and G. J. Jellis, eds. Blackwell Scientific Publications, Oxford.

9. Castanho, B., and Butler, E. E. 1978. Rhizoctonia decline: Studies on hypovirulence and potential use in biological control. Phytopathology 68:1511-1514 
10. Castanho, B., Butler, E. E., and Shepherd, R. J. 1978. The association of double-stranded RNA with Rhizoctonia decline. Phytopathology 68:1515-1519.

11. Chen, B., Choi, G. H., and Nuss, D. L. 1993. Mitotic stability and nuclear inheritance of integrated viral cDNA in engineered hypovirulent strains of the chestnut blight fungus. EMBO (Eur. Mol. Biol. Organ.) J. 12:2991-2998

12. Choi, G. H., and Nuss, D. 1992. Hypovirulence of chestnut blight fungus conferred by an infectious viral cDNA. Science 257:800-803.

13. Dodds, J. A. 1980. Association of type 1 viral-like dsRNA with clubshaped particles in hypovirulent strains of Endothia parasitica. Virology 107:1-12.

14. Elliston, J. E. 1982. Hypovirulence. Pages 1-33 in: Advances in Plant Pathology. Vol. 1. D. S. Ingram and P. H. Williams, eds. Academic Press Ltd., London.

15. Enebak, S. A., MacDonald, W. L., and Hillman, B. I. 1994. Effect of dsRNA associated with isolates of Cryphonectria parasitica from the central Appalachians and their relatedness to other dsRNAs from North America and Europe. Phytopathology 84:528-534.

16. Fenstermacher, J. M. 1979. Certain features of dollar spot disease and its causal organism, Sclerotinia homoeocarpa. Pages 49-53 in: Advances in Turfgrass Pathology. P. O. Larsen and B. J. Joyner, eds. Harcourt Brace Jovaniovich, Duluth, MN.

17. Fulbright, D. W. 1984. Effect of eliminating dsRNA in hypovirulent Endothia parasitica. Phytopathology 74:722-724.

18. Goodman, D. M., and Burpee, L. L. 1991. Biological control of dollar spot disease of creeping bentgrass. Phytopathology 81:1438-1446.

19. Hammar, S., Fulbright, D. W., and Adams, G. C. 1989. Association of double-stranded RNA with low virulence in an isolate of Leucostoma persoonii. Phytopathology 79:568-572.

20. Hillman, B. I., Fulbright, D. W., Nuss, D. L., and van Alfen, N. K. 1995. Hypoviridae. Pages 261-264 in: Virus Taxonomy: Sixth Report of the International Committee for the Taxonomy of Viruses. F. A. Murphy, C. M. Fauquet, D. H. L. Bishop, S. A. Ghabrial, A. W. Jarvis, G. P. Martell, M. P. Mayo, and M. D. Summers, eds. Springer-Verlag, New York.

21. Hillman, B. I., Tian, Y., Bedker, P. J., and Brown, M. P. 1992. A North American hypovirulent isolate of the chestnut blight fungus with European isolate-related dsRNA. J. Gen. Virol. 73:681-686.

22. Howitt, R. L. J., Beever, R. E., Pearson, M. N., and Forster, L. S. 1995. Presence of double-stranded RNA and virus-like particles in Botrytis cinerea. Mycol. Res. 99:1472-1478.

23. Kousik, C. S., Snow, J. P., and Valverde, R. A. 1994. Comparison of double-stranded RNA components and virulence among isolates of Rhizoctonia solani AG-1 IA and AG-1 IB. Phytopathology 84:44-49.

24. L'Hostis, B., Hiremath, S. T., Rhoads, R. E., and Ghabrial, S. A. 1985. Lack of sequence homology between dsRNA from European and American hypovirulent strains of Endothia parasitica. J. Gen. Virol. 66:351-355.

25. Liang, P., Chen, K., and Liu, H. 1987. Virus particles in sporophore of oyster mushroom. Chin. J. Virol. 3:370-375.

26. MacDonald, W. L., and Fulbright, D. W. 1991. Biological control of chestnut blight: Use and limitations of transmissible hypovirulence.
TABLE 4. Comparison of virulence of subcultures of Sclerotinia homoeocarpa after curing treatments of heat and cycloheximide

\begin{tabular}{lcc}
\hline Isolate $(\text { treatment })^{\mathrm{x}}$ & Length of lesion $^{\mathrm{y}}(\mathrm{mm})$ & dsRNA $^{\mathrm{z}}$ \\
\hline Sh48B $\left(0 \mu \mathrm{g} / \mathrm{ml}, 20^{\circ} \mathrm{C}\right)$ & $23 \mathrm{a}$ & - \\
Sh12B-C1 $\left(20 \mu \mathrm{g} / \mathrm{ml}, 30^{\circ} \mathrm{C}\right)$ & $23 \mathrm{a}$ & - \\
Sh12B-C2 $\left(20 \mu \mathrm{g} / \mathrm{ml}, 28^{\circ} \mathrm{C}\right)$ & $22 \mathrm{a}$ & - \\
Sh12B-C4 $\left(10 \mu \mathrm{g} / \mathrm{ml}, 30^{\circ} \mathrm{C}\right)$ & $22 \mathrm{a}$ & - \\
Sh12B-C3 $\left(15 \mu \mathrm{g} / \mathrm{ml}, 30^{\circ} \mathrm{C}\right)$ & $20 \mathrm{a}$ & - \\
Sh12B $\left(0 \mu \mathrm{g} / \mathrm{ml}, 20^{\circ} \mathrm{C}\right)$ & $2 \mathrm{~b}$ & + \\
PDA & 0 & \\
\hline
\end{tabular}

${ }^{\mathrm{x}}$ Isolates were treated with various concentrations of cycloheximide and incubated at different temperatures for 2 weeks. Hyphal tip subcultures from the treated colonies were used in the virulence test. PDA indicates sterile potato dextrose agar plugs.

y Virulence was evaluated on detached leaves of creeping bentgrass and length of lesions was measured 3 days after inoculation. Means followed with the same letter in the column are not significantly different according to Dunnett's $t$ test $(P=0.05)$.

${ }^{\mathrm{z}}$ Presence (+) and absence (-) of dsRNA were detected using hyphal tip subcultures grown on PDA.

\section{Plant Dis. 75:656-661.}

27. Melzer, M. S., and Boland, G. J. 1995. Transmissible hypovirulence in Sclerotinia minor. Can. J. Plant Pathol. 18:19-28.

28. Morris, T. J., and Dodds, J. A. 1979. Isolation and analysis of doublestranded RNA from virus-infected plant and fungal tissue. Phytopathology 69:854-858.

29. Nelson, E. B., and Craft, C. M. 1991. Introduction and establishment of strains of Enterobacter cloacae in golf course turf for the biological control of dollar spot. Plant Dis. 75:510-514.

30. Nuss, D. L., and Koltin, Y. 1990. Significance of dsRNA genetic elements in plant pathogenic fungi. Annu. Rev. Phytopathol. 28:37-58.

31. Paul, C. P., and Fulbright, D. W. 1988. Double-stranded RNA molecules from Michigan hypovirulent isolates of Endothia parasitica vary in size and sequence homology. Phytopathology 78:751-755.

32. Polashock, J. J., Anagnostakis, S. L., Milgroom, M. G., and Hillman, B. I. 1994. Isolation and characterization of a virus-resistant mutant of Cryphonectria parasitica. Curr. Genet. 26:528-534.

33. Smiley, R. W. 1983. Compendium of Turfgrass Diseases. The American Phytopathological Society, St. Paul, MN.

34. Vargas, J. M. 1981. Management of Turfgrass Diseases. Burgess Publishing Co., Minneapolis, MN.

35. Yelton, M. M., Hamer, J. E., and Timberlake, W. E. 1984. Transformation of Aspergillus nidulans by using a trpC plasmid. Proc. Natl. Acad. Sci. U.S.A. 81:1470-1474.

36. Zhang, R., Dickinson, M. J., and Pryor, A. 1994. Double-stranded RNAs in the rust fungi. Annu. Rev. Phytopathol. 32:115-133.

37. Zhou, T., and Boland, G. J. 1993. Hypovirulence and double-stranded RNA in Sclerotinia homoeocarpa. (Abstr.) Phytopathology 83:1347. 